\title{
O PERFIL DOS USUÁRIOS CADASTRADOS NA ASSOCIAÇÃO PARANAENSE DE OSTOMIZADOS - APO*
}

\author{
M aria de Fátima M antovani ${ }^{1}, \mathrm{M}$ arcio Roberto $M$ uniz $^{2}, \mathrm{M}$ ariana Cavalcanti Simões ${ }^{3}, \mathrm{M}$ arta Daniela B oschco ${ }^{4}$, \\ Gabrielle Delattre de Oliveira ${ }^{5}$
}

RESUM O: Trata-se de um estudo quantitativo real izado no período de setembro de 2005 a janeiro de 2006. 0 objetivo foi caracterizar os portadores de ostomias, cadastrados na A ssociação Paranaense dos Ostomizados (A PO). Foram analisados 1538 prontuários da APO e destes foram selecionados 477 com dados completos. Em relação ao sexo, 53,7\% dos ostomizados eram do sexo masculino e 46,3\% do feminino. Quanto ao motivo da intervenção cirúrgica, na faixa etária de 0 a 45 anos, 0 estudo aponta para as causas externas como uma implicação para a realização das ostomias, representando $20 \%$ do total de intervenções realizadas. Entretanto na faixa etária acima de 45 anos, 51,4\% desta população eram do sexo feminino, sendo que, as neoplasias intestinais foram responsáveis pela concretização de aproximadamente $51 \%$ das ostomias. A s colostomias representam $65,4 \%$ dos procedimentos real izados. R ecomendase a continuação do estudo com intuito de contribuir na identificação das necessidades educativas da clientela.

DESCRITORES: Ostomia; Cuidados de Enfermagem.

\section{MEMBERS' PROFILE REGISTERED IN OSTOMATE ASSOCIATION OF PARANÁ - OAP}

\begin{abstract}
It is a quantitative study performed from September/2005 to January/2006. The objective was to characterize the ostomates registered at the Ostomate A ssociation of Paraná State (APO in Portuguese). From 1538 analyzed forms, 477 were selected with complete data. A ccording to sex, $53.7 \%$ of the ostomates were male, and $46.3 \%$ were female; from 0 to 45 years old, there was predominance of male patients, $63.9 \%$. A s for the reasons of the surgical procedure, at the age of 0 to 45 years old, the study points out external causes as an implication for the ostomy procedure, standing for $20 \%$ of the total surgeries. However, at ages above 45 years old, $51.4 \%$ of the population was female, and intestinal neoplasm was responsible for about $51 \%$ of the ostomies. Colostomies accounted for $65.4 \%$ of the procedures. It is suggested the study continuation in order to contribute for the identification of ostomates' educational needs.
\end{abstract}

DESCRIPTORS: Ostomy; N urse's care.

\section{EL PERFIL DE LOS USUARIOS CON CATASTRO EN LA ASOCIACIÓN PARANAENSE DE OSTOMIZADOS - APO}

RESUMEN: Este es un estudio cuantitativo real izado en el periodo de septiembre de 2005 a enero de 2006. El objetivo fue caracterizar los portadores de ostomias, con catastro en la A sociación Paranaense de los O stomizados (A PO). Fueron analizados 1538 prontuarios de la APO y de estes fueron el ejidos 477 con datos completos. En relacón al sexo, 53,7\% dos "ostomizados" eran del masculino y $46,3 \%$ del femenino. Cuanto al motivo de la intervención quirúrgica, en la franja etaria de 0 a 45 años, el estudio apunta para las causas externas como una implicación para la realización de las ostomias, lo que representa $20 \%$ del total de intervenciones realizadas. Sin embargo en la franja etaria de más de 45 años, 51,4\% de esta población era del sexo femenino, siendo las neoplasias intestinales responsables por la concretización de aproximadamente $51 \%$ de las ostomías. L as col ostomías representam $65,4 \%$ de los procedimientos realizados. Se recomienda continuación del estudio a fin de contribuir en la identificación de las necesidades educativas de la clientela. DESCRIPTORES: Ostomias; Cuidados de enfermería.

\footnotetext{
*Pesquisa vinculada ao Projeto de Extensão "A ssistindo o A dulto com Ostomia", da Pró-R eitoria de Extensão e Cultura da Universidade Federal do Paraná - UFPR.

${ }^{1}$ Enfermeira. Doutora. Professora do Departamento de Enfermagem da UFPR. Coordenadora do Projeto.

${ }^{2}$ A cadêmico do Curso de Enfermagem da UFPR. Bolsista de Extensão da PROEC.

${ }^{3}$ A cadêmica do Curso de Enfermagem da UFPR. Bolsista de Extensão da PROEC.

${ }^{4}$ A cadêmica do Curso de Enfermagem da UFPR. Bolsista de Extensão da PROEC.

${ }^{5}$ A cadêmica do Curso de Enfermagem da UFPR.
}

A utor correspondente:

$M$ aria de Fátima M antovani

$\begin{array}{ll}\text { R. Rio Iriri, } 20 \text { bloco 01, ap. 21- 82840-310 - Curitiba-PR } & \text { Recebido em: 20/02/07 }\end{array}$

E-mail: mantovan@ufpr.br 


\section{INTRODUÇÃO}

0 Projeto de Extensão "A ssistindo o A dulto com Ostomia", do D epartamento de Enfermagem, da U niversidade Federal do Paraná, real izado pelo G rupo de Estudos Multiprofissional em Saúde do A dulto (GEMSA), atuou durante os anos de 2001 a 2005, com à A ssociação Paranaense dos Ostomizados (APO). N este período, identificou e caracterizou os problemas apresentados pelos portadores de ostomias, bem como participou do atendimento e orientação a esta clientela. A intenção de desenvolver este trabal ho surgiu por não existir conhecimento acerca das características dos freqüentadores da A PO, que buscavam informações após a cirurgia quanto aos cuidados relacionados as ostomias.

As associações, dentro do processo de reinclusão e suporte social, mostram-se extremamente importantes, além de atividades educativas com os pacientes e familiares no tocante aos cuidados dispensados às ostomias, promovem encontros periódicos, visando à troca de experiências e confraternização entre os ostomizados. N esse sentido, ressaltamos que o estabelecimento de vínculos interpessoais, grupais ou comunitários próximos, proporcionam um sentimento de proteção e apoio às pessoas envolvidas ${ }^{(1)}$.

A s associações de ostomizados são entidades sem fins lucrativos, que contam com a colaboração de pessoas voluntárias na realização das atividades e estão presentes em todo o território nacional. Estas têm por finalidade, mediante a participação destes indivíduos, principalmente do paciente ostomizado, garantir e reivindicar os direitos básicos desta clientela, portanto, o caráter social está fortemente atrelado à história destas organizações.

Deste modo, os associados, principalmente os recém ostomizados, percebem a existência de várias pessoas que vivem nas mesmas condições, e que exercem suas atividades diárias com desenvoltura. As A ssociações são elementos fundamentais apontados pela literatura como fontes essenciais de suporte ao processo de reabilitação dos ostomizados(2) e representam um novo espaço social para as pessoas que fizeram a cirurgia para construção da ostomia ${ }^{(3)}$.

A palavra ostomia pode ser definida como uma abertura confeccionada para exteriorizar uma víscera oca através do corpo ${ }^{(2)}$. No caso dos associados da APO, são freqüentemente ostomias intestinais e urinárias.
As ostomias são divididas em temporárias e permanentes ${ }^{(2)}$, sendo que, neste caso específico, os nomes a elas atribuídos devem-se à parte do intestino ou do aparelho urinário que foi exteriorizado no abdômen. A s ostomias temporárias têm a finalidade de alívio do volume de urina ou fezes, acumulados na bexiga ou intestino, até que o paciente possa vir a excretá-las normalmente. Porém, em casos que, por algum motivo, uma dessas vias é lesionada de maneira irreversível, adota-se o desvio permanente, como forma de garantir a filtração renal, bem como 0 funcionamento do intestino e, conseqüentemente, a formação da urina ou das fezes.

Estas cirurgias encontram-se no plano terapêutico de muitas enfermidades que, na maioria das vezes, são situações clínicas vinculadas a doenças crônicas, intestinais e urinárias, como os cânceres e as doenças inflamatórias além de traumas e enfermidades congênitas ${ }^{(4)}$.

Nos últimos anos, houve um crescente número de ostomias temporárias realizadas devido a traumas decorrentes de ferimentos por arma de fogo (FAF) e ferimentos por arma branca (FAB ), visto que é notória a incidência deste tipo de ocorrência em meio à população. De acordo com os dados do Sistema Integrado de A tendimento ao Trauma e Emergência (SIATE) em Curitiba(5), no ano de 2002 foram atendidas pelas ambulâncias do Corpo de B ombeiros 1269 vítimas de FAF e FA B na cidade de Curitiba e R egião M etropolitana. N ão obstante, no ano seguinte, o número de vítimas cresceu significativamente, foram 1467 ocorrências desta natureza.

Outro fator desencadeante de ostomias intestinais é o câncer colo-retal. A estimativa para 0 ano de 2005 no Brasil apontava esse como o quarto tumor maligno mais freqüente para ambos os sexos. A maioria dos casos de câncer de intestino ocorre na faixa etária entre 50 e 70 anos, mas as possibilidades de desenvolvimento já aumentam a partir dos 40 anos ocorrendo maior incidência em portadores de doenças inflamatórias intestinais ${ }^{(6)}$. Devido à sua cronicidade, doenças inflamatórias intestinais como a retocolite ulcerativa e a doença de C rohn favorecem a manifestação de neoplasias na região de cólon e reto ${ }^{(7)}$

A vinculação da ostomia a doenças crônicas, cânceres e traumas acaba por afastar, no entendimento de pessoas leigas, este procedimento do âmbito pediátrico, porém, este processo é bastante comum com as crianças. Neste sentido, 
a abertura de ostomas é utilizada, rotineiramente, no tratamento de uma série de doenças cirúrgicas da criança e em grande variedade de situações clínicas. Felizmente, como é utilizada, praticamente, apenas no tratamento de doenças benignas, os ostomas em crianças têm como uma de suas principais características o caráter temporário(2:69).

Em face do exposto, propusemos a caracterização do perfil do ostomizado cadastrado na A PO. Pois, além da relevância desta temática, observamos que, durante a realização do Projeto de Extensão, muitos dados estavam se perdendo, devido à forma de armazenamento na instituição, que presta serviços à comunidade desde o ano de 1989. Vimos nesta intervenção a possibilidade de conhecer os aspectos relativos desta realidade, bem como contribuir para o levantamento das características da população cadastrada na A PO.

\section{METODOLOGIA}

A pesquisa tem uma abordagem quantitativa descritiva. Os dados foram coletados por intermédio de uma pesquisa documental, sendo que o instrumento de coleta aplicado foi um questionário baseado nos prontuários dos pacientes cadastrados na A ssociação Paranaense dos Ostomizados. 0 período da coleta concentrou-se entre os meses de setembro de 2005 a janeiro de 2006.

0 número de pacientes cadastrados do ano de 1989, data da fundação da A PO, até janeiro de 2006, data do término da coleta de dados, foi de 1538 . No entanto, apenas 477 prontuários fizeram parte da amostra, pois foram excluídos os prontuários com dados incompletos a respeito do tempo e do motivo da realização da ostomia, seu tipo, caráter e dados de identificação.

0 projeto de pesquisa foi apresentado ao Comitê de Ética em Pesquisa do Setor de Ciências da Saúde da Universidade Federal do Paraná, sendo aprovado no dia 09 de novembro de 2005, sob 0 número 224SM 100.05.11, por atender os princípios éticos estabelecidos pela Resolução 196/96: autonomia - consentimento livre e esclarecido dos participantes e proteção dos grupos vulneráveis; beneficência - compromisso em proporcionar 0 máximo de benefícios e o mínimo de danos; não mal eficência - garantia de que prováveis danos serão evitados e justiça e eqüidade, relevância social para os sujeitos e minimização do ônus para sujeitos vulneráveis ${ }^{(8)}$.

A pós a seleção de dados válidos, os mesmos foram organizados e analisados em tabelas e apresentados através de planilhas e gráficos do programa Excell.

\section{APRESENTAÇÃO E ANÁLISE DOSDADOS}

Os dados são apresentados e analisados utilizando-se os parâmetros sexo e idade com o intuito de permitir uma mel hor comparação das variáveis em análise. Em um primeiro momento, foi realizado um levantamento do local de residência da clientela da A PO, Tabela 1.

Tabela 1 - Caracterização dos freqüentadores de acordo com o local de residência, idade e sexo. Curitiba, 2006

\begin{tabular}{llccccc}
\hline $\begin{array}{l}\text { Local residência } \\
\text { idade/sexo }\end{array}$ & & Curitiba & Reg. Metropolitana & Outras Cidades PR & Outros Estados & Total \\
\hline $0-12$ & Mas & 4 & 2 & 1 & - & - \\
& Fem & 1 & - & - & - & 1 \\
$13-19$ & Mas & 4 & 5 & - & - & 9 \\
& Fem & 3 & - & - & 2 & 3 \\
$20-45$ & Mas & 48 & 30 & 4 & - & 85 \\
& Fem & 23 & 25 & 5 & - & 53 \\
$46-60$ & Mas & 46 & 21 & 10 & - & 75 \\
& Fem & 45 & 34 & 10 & 1 & 89 \\
mas de 61 & Mas & 57 & 12 & 1 & - & 75 \\
\hline Total & Fem & 55 & 18 & $\mathbf{3 9}$ & & $\mathbf{3}$ \\
\hline
\end{tabular}


Os números, apresentados na Tabela 1 , demonstram que os usuários da A ssociação Paranaense de Ostomizados estão concentrados na Capital do Estado e região metropolitana. Este fato pode ser explicado pela localização da APO em Curitiba, bem como, pela descentralização da distribuição das órteses, em 01 de outubro de $2001^{(9)}$, e pela criação de núcleos, pequenas associações, em outras regiões do Estado.

A apreciação dos dados da Tabela 2 aponta para certa homogeneidade na distribuição dos números de ostomias em relação ao sexo, isto é, $53,7 \%$ ostomizados do sexo masculino frente a $46,3 \%$ ostomizados do sexo feminino. Contudo, há uma discrepância quando se relaciona o sexo com a idade em que ocorreram as intervenções cirúrgicas. $\mathrm{Na}$ faixa etária de 0 a 45 anos, 63,9\% dos ostomizados eram do sexo masculino, entretanto, pode-se supor que esta diferença possa ser explicada ao se analisar as causas que levam à ostomia. A ocorrência de ostomias por causas externas é expressiva no sexo masculino, principalmente, entre a faixa etária de 0 a 45 anos.

Do total de procedimentos realizados nesta faixa etária, cerca de $20 \%$ tiveram como causa 0 trauma, sendo que destes pacientes, aproximadamente $87 \%$ eram do sexo masculino. Quando se comparam estes números com os dados de mortal idade e morbidade, devido à causas externas ocorridas em via pública, fornecidos pelo DATA SUS, ratifica-se esta questão, isto é, dos 35.546 óbitos por causas externas no ano de $2004^{(10)}$, na faixa etária de
0 a 49 anos, 89,5\% eram do sexo masculino. No tocante à morbidade hospital ar, dos 48.037 pacientes internados pelo SUS, no ano de 2006, pelo mesmo motivo(11), 75,33\% eram do sexo masculino.

$\mathrm{Na}$ idade acima de 45 anos, ainda relacionando com o sexo do paciente, os dados voltam a ficar homogêneos com $48,6 \%$ ostomizados do sexo masculino, porém, cabe ressaltar que há uma inversão quanto à população mais afetada, isto é, $51,4 \%$ dos ostomizados são do sexo feminino. Pode-se supor que tal fato ocorra pela maior incidência do câncer colo-retal no sexo feminino, ou ainda pela ocorrência de outros tipos de neoplasias específicas da mulher que possam levar, mesmo que de forma indireta, a realização de uma ostomia. 0 câncer de colo uterino, quando do seu tratamento através da braquiterapia, pode, de forma secundária, vir a lesionar as vias intestinais ou urinárias, tornando necessária a concretização de uma ostomia. Os efeitos tardios do tratamento incluem as retites e as cistites crônicas, as fistulas vésico-vaginais e reto-vaginais, e os quadros de enterite com graus variáveis de obstrução intestinal ${ }^{(6)}$.

Levando-se em consideração apenas a faixa etária da amostra, observa-se que a maior incidência de ostomias ocorre a partir dos 45 anos. Dos 477 prontuários anal isados, $66,9 \%$ encontravam-se nesta faixa etária, sendo que $51,4 \%$ desta clientela tiveram como causa da realização da ostomia neoplasias intestinais, o que aponta para o provável motivo do maior índice de ostomizados.

Tabela 2 - Caracterização dos freqüentadores de acordo com a causa que levou a ostomia, idade e sexo. Curitiba, 2006

\begin{tabular}{lcccccccc}
\hline $\begin{array}{l}\text { Causas } \\
\text { idade/sexo }\end{array}$ & & $\begin{array}{c}\text { Neoplasias } \\
\text { Intestinais }\end{array}$ & $\begin{array}{c}\text { Outras } \\
\text { Neoplasias }\end{array}$ & $\begin{array}{c}\text { Causas } \\
\text { Externas }\end{array}$ & $\begin{array}{c}\text { Outras Doenças } \\
\text { Crônicas }\end{array}$ & $\begin{array}{c}\text { Outras } \\
\text { Causas }\end{array}$ & $\begin{array}{c}\text { Não } \\
\text { Identificado }\end{array}$ & Total \\
\hline $0-12$ & Mas & - & - & - & 6 & - & 1 & 7 \\
\multirow{2}{*}{$13-19$} & Fem & - & - & 1 & - & - & - & 1 \\
& Mas & - & - & 5 & 4 & - & - & 9 \\
$20-45$ & Fem & 1 & - & 2 & - & - & - & 3 \\
& Mas & 18 & 2 & 22 & 16 & 18 & 9 & 85 \\
\multirow{2}{*}{$46-60$} & Fem & 29 & 8 & 1 & 4 & 4 & 7 & 53 \\
\multirow{2}{*}{ mais de 61} & Mas & 36 & 5 & 1 & 8 & 17 & 8 & 75 \\
& Fem & 41 & 14 & 1 & 8 & 20 & 5 & 89 \\
Total & Mas & 48 & 13 & 2 & 4 & 11 & 2 & 80 \\
\hline
\end{tabular}


Tabela 3 - Caracterização dos freqüentadores de acordo com o tipo de ostomia, idade e sexo. Curitiba, 2006

\begin{tabular}{llcccccc}
\hline $\begin{array}{l}\text { Causas } \\
\text { idade/sexo }\end{array}$ & Colostomia & Urostomia & Ileostomia & Outros & $\begin{array}{c}\text { Não } \\
\text { Identificado }\end{array}$ & Total \\
\hline $0-12$ & Mas & 5 & 1 & - & - & 1 & 7 \\
\multirow{2}{*}{$13-19$} & Fem & 1 & - & - & - & - & 1 \\
& Mas & 4 & - & 1 & 1 & 3 & 9 \\
$20-45$ & Fem & 2 & - & 1 & - & - & 3 \\
& Mas & 53 & 1 & 16 & 4 & 11 & 85 \\
$46-60$ & Fem & 36 & 4 & 8 & - & 5 & 53 \\
& Mas & 45 & 5 & 15 & 3 & 7 & 75 \\
mais de 61 & Fem & 61 & 6 & 9 & 3 & 10 & 89 \\
& Mas & 50 & 9 & 11 & 4 & 6 & 80 \\
Total & Fem & 55 & 6 & 7 & 2 & 5 & 75 \\
\hline
\end{tabular}

Quando se analisa a Tabela 3, no tocante ao tipo predominante de ostomia, observa-se que a colostomia é a mais freqüente, $65,4 \%$ dos procedimentos realizados na população considerada e, $66,1 \%$ nos pacientes acima de 45 anos. Estes dados corroboram com a literatura, bem como com os dados apresentados na Tabela 2, que apontam uma maior propensão desta população para desenvolver neoplasias intestinais. Desta forma ressal tamos que,

as estimativas de incidência de câncer no Brasil para 2006 apontam o câncer colo-retal como o 50 tumor maligno mais freqüente entre homens (com 11.390 casos novos) e $4^{\circ}$ entre as mulheres ( 13.970 casos novos). A maior incidência de casos ocorre na faixa etária entre 50 e 70 anos, mas as possibilidades de desenvolvimento já aumentam a partir dos 40 anos $^{(12)}$.

É importante salientar que neste estudo houve a constatação de um caso de neoplasia intestinal na faixa etária de 13 a 19 anos. Mesmo tratando-se de um caso, este dado é bastante significativo em face do apresentado na literatura quanto à incidência deste tipo de neoplasia.

\section{CONSIDERAÇÕES FINAIS}

Este trabalho proporcionou ampliar o conhecimento quanto ao perfil dos usuários da A ssociação Paranaense de Ostomizados, além de apontar deficiências nos registros desta instituição, visto que, muitos prontuários destes pacientes, estavam incompletos, inviabilizando o seu uso durante a coleta e análise de dados.

A partir desta experiência, propomos que sejam elaborados outros trabalhos desta natureza, com 0 intuito de contribuir na identificação das necessidades de cuidados desta clientela, bem como, modificações nos prontuários dos associados. Isto possibilitará 0 desenvolvimento de futuras pesquisas, viabilizando o apontamento de meios para uma intervenção significativa a esta clientela. Porquanto essas informações poderão auxiliar o profissional de saúde, em especial o enfermeiro, com parâmetros para realizar atividades educativas a fim de diminuir complicações vinculadas a falta de orientação. Há ainda, a possibilidade de melhorar, mediante a realização de outros estudos, a qualidade de vida de pessoas portadoras dessa condição de saúde.

\section{REFERÊNCIAS}

1. Campos EP. Quem cuida do cuidador: uma proposta para os profissionais da saúde. Petrópolis: Vozes; 2005.

2. Santos VLCG, Cesaretti IUR. A ssistência em estomaterapia: cuidando do ostomizado. São Paulo: A theneu; 2000.

3. Mantovani M F. Sobrevivendo: o significado do adoecimento e o sentido da vida pós-ostomia [tese]. São Paulo (SP): Escola de Enfermagem da Universidade de São Paulo; 2001.

4. Osório F. Indicações para ostomias digestivas. Rev Liga Ostom Portugal 1999: 19.

5. Prefeitura Municipal de Curitiba. SIATE Sistema integrado de atendimento ao trauma e emergência. Estatísticas, Secretaria M unicipal de Saúde: Curitiba; 2005. 
6. M inistério da Saúde (BR). Programa de Qual idade em Radioterapia - Primeiro Seminário em Radioterapia. Instituto Nacional do Câncer [acesso em $2007 \mathrm{~F} \mathrm{ev}$ 09]. Disponível em: www.inca.gov.br/pgrt/download/tec_int

7. Tavares A. Órgão divulgador daA ssociação B rasileira de Ostomizados. Rev A brasco 2005; (5):14-5.

8. M inistério da Saúde (BR). Conselho Nacional de Saúde. Resolução n.196 de 10 de outubro de 1996. Dispõe sobre as diretrizes e normas regulamentadoras de pesquisas envolvendo seres humanos [acesso 2006 Out 09]. Disponível em: www.saudeufpr.br

9. Prefeitura M unicipal de Curitiba. M emorando 216/ 2001, de 01 de outubro de 2001. Dispõe sobre as normas adotadas para dispensação de bolsas de colostomia eurostomia para usuários do SU S/C uritiba. Secretaria M unicipal de Saúde, Curitiba, 01 out. 2001.

10. M inistério da Saúde (BR). M ortalidade por Causas Externas Ocorridas em Via Pública por Local de Residência [acesso em 2007 Jan 30]. Disponível em: www.datasus.gov.br

11. M inistério da Saúde (BR). M orbidade Hospitalar do SUS por Causas Externas Ocorridas em Via Pública por Local de Residência [acesso em 2007 Jan 30]. Disponível em: www.datasus.gov.br.

12. M inistério da Saúde (BR). Instituto N acional do Câncer [acesso em 2005 Ago 17]. Disponível em: www.inca.gov.br 